\title{
Detection of Tumor Cells in Brain using Cellular Automata with Image Segmentation and Edge Detection.
}

\author{
Jasmeena Tariq, A. Kumaravel
}

\begin{abstract}
Tumor growth or, growth of cancerous cells is a big challenge in today's medical word. When dealing with human life, the detection of tumors through computers has to be highly accurate. Thus we require the assistance of computer in medical examinations, so that we will get very low rate of false cases. Brain tumor, in today's world, is seen as most threatening and life taking disease. In order to detect brain tumor more accurately in lesser time, many techniques have already been proposed using image segmentation and edge detection. In our paper we propose a technique which is more efficient to detect brain tumor where edge detection through cellular automata have been used from Magnetic Resonance Imaging (MRI) scan images. It processes these images, and determines the area affected by using segmentation and edge detection with cellular automata. Simulated work is completed with the help of Simulink in MATLAB. Regarding this particular topic there are many studies, however our proposal of combination of both segmentation and edge detection through cellular automata shows better results as compared to combining segmentation with classical edge detection in term of computation time and clarity. This will help in efficiency of detecting brain tumor and later in its removal.
\end{abstract}

Keywords: Brain Tumor, Morphological Operations MRI, Watershed Segmentation, MATLAB.

\section{INTRODUCTION:}

Human body is made up of cells. Each cell in a human body has its own particular function. They grow in particular order for correct functioning of body. Tumor (a mass of excessive tissue) is the result of unwanted rapid growth of cells, creating a dire situation for a person.In medical world, the innovation of Imaging technology helped surgeons in visualizing the parts into the body for precise and exact diagnosis. It provided a way for the surgeons to create surgeries into the body precisely while not cutting and opening excessive amount of the body. MRI (Magnetic Resonance Imaging) gave better results than X-Ray imaging, as an MRI shows a person's interior body portions in 3D. Here, we can make use of Image processing algorithms, to make it easy for doctors to analyse the symptoms of patients, because the pictures generated by using MRI are further enhanced using image processing. Concept of Cellular automata was discovered in early 1940's by John V. Neumann and Stanislaw Ulam.

Revised Manuscript Received on November 05, 2019.

Dr Kumaravel*, Professor and Dean, School of Computing, Bharath University, Chennai.

Dr Kumaravel, Professor and Dean, School of Computing, Bharath University, Chennai.

(C) The Authors. Published by Blue Eyes Intelligence Engineering and Sciences Publication (BEIESP). This is an open access article under the CC BY-NC-ND license (http://creativecommons.org/licenses/by-nc-nd/4.0/)
J. V. Neumann gave the concept of universality in cellular automaton. According to his description of CA, each cell has/represents a state-space of twenty- nine states, and it can execute every possible computable operation. Many researchers have used cellular automata (CA) since 1940s. Cellular automata is used in many fields like the models having biological perspective, cryptography, simulation, language recognition, image processing, computer architecture and many such and other fields.

In last ten years, researchers have done much work in the field of CA with respect to edge detection in an acquired image. In Edge detection, agents used are local and hence the edge maps which are obtained as an output image can be fragmented, as the edges in the input image will not be always locally distinct. Image pixels in an image define an edge. If the intensity of an image changes abruptly, it gives rise to an edge. An edge pixel occurs due to change in the intensity of picture pixel.

\section{EDGE DETECTION}

It is the process of identifying and finding sharp discontinuities in a picture. The pixels which are at the boundary of an image show abrupt changes between them, giving rise to discontinuity. The classical edge detection methods are sensitive to giant gradients within the image and returned zero in those regions of an image which are uniform.

\section{RELEVANT WORK}

In edge detection many methods have been given by the researchers over few decades. Each one of those methods has its own advantages and disadvantages. Much of the work has been done in this field and it has been beneficial in so many applications like image recognition, enhancement, morphing of an image, image compression etc.

Zubair Jeelani et al.[1] proposed an algorithm for detection and removal of noise in an image.

FaselQadir et al. [2] has proposed the classification of three edge detection rules such as no-edge, sturdy edge and weak edge detection through thorough investigation of linear rules used in CA. The results shown, by comparing it with various other already given techniques, were fascinating with regards to its performance.

Shohei Sato et al. [3] proposed the cellular automata based edge detecting technique which was more efficient than it previous techniques.

Blue Eyes Intelligence Engineering 
KhadijehMirzaei et al. [4] has used fuzzy CA and proposed a method for edge detection and noise elimination in an image. The algorithm proposed works on images with salt and pepper noise thus eliminating it and takes gray-level. image and detects edges.

Parwinder Kaur Dhillon [5] again used CA for edge detection in an image, dividing image in edge-pixels and non edge-pixels. The algorithm proposed is more efficient. and gives values to the edges based on the already acquired division of pixels.

Haralick[10] proposed second directional spinoff edge detector. It is considered a hybrid of testing technique which uses applied math and differentiation technique, that results in higher performance during a noisy setting of an environment.

There always appears a problem in proposing an edge detection algorithm because of noise, external interference, some of the most needed edges are missing, edge detection can be false sometimes etc. As already proposed by FaselQadir et al. [2] we classify the linear rules of CA which will be used for edge detection. Our idea is more. efficient because in our algorithm we add a periodic boundary to the image so that the edges will be more clear in difficult images. After doing a comparative study, our algorithm is more efficient than its previous one.

\section{CELLULAR AUTOMATA}

\section{1:- Edge Detection using Cellular Automata:-}

We can apply rules(1D and 2D) on images based on their dimension for edge detection, but in our algorithm we use two-dimensional CA. In edge detection there are many steps involved and it is shown in figure below.

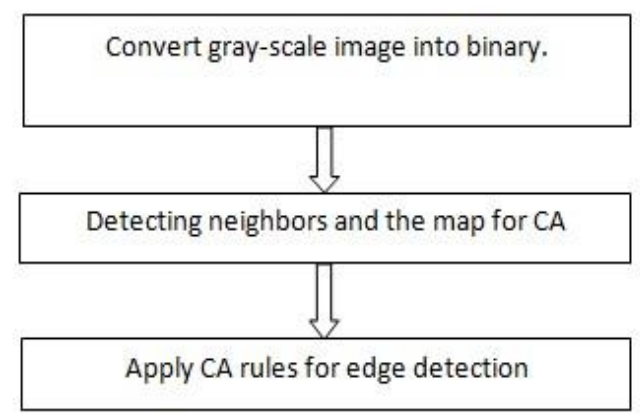

4.2:- Binary image conversion from grey scale image: Our algorithm gets an input image from MRI machine and will first convert gray-scale image into its binary one. The binary image which we get as an output is represented by an array, which can take values 0 's(for white) and 1's(for black). The toolbox function im2bw() is used in MATLAB. Among various methods available, threshold method is used in our study. We choose a threshold value, V. We label the pixels (different values for back-ground "a" and object "b") automatically, and use it, where $a=0$ (white) and $b=1$ (black).

\section{3:- Setting neighbours and applying CA rules:}

\subsection{1:- Setting neighbours:-}

After setting the neighbouring cells we apply Cellular automata rule 124 , where the state of a cell is dependent upon its neighbourhood cells, and its present state. The set of states in our algorithm is represented by $\mathrm{S}$.

$\mathrm{S}=\{0,1\}$. Dead cell $=0$, Alive cell $=1$.

A is a set of position of neighbour cells, the new state of this cell is computed by $\left(\sum \max A=9\left\{9^{*} 1\right\}\right.$ and $\sum \min$ $\mathrm{A}=0\{0 * 1\}$ )

New cell state $=\left(a_{-1} a_{1}\right)+\left(a_{-1} a_{0}\right)+\left(a_{-1} a_{0}\right)$

\subsection{2:- CA Rules applied here: -}

Rules to detect brain tumor by applying edge detection with CA requires few steps. We need a rule which will prove to be efficient for edge detection in images, this has been done by applying Wolfram's rule no. 124. We have applied it for many images and the output was quite efficient and accurate.

Numbers of Decision rules are taken as:

Total no. of states $=2$, if the value is " 0 " the cell is considered as dead, for the value of " 1 " the cell is considered as live.

Total no. of neighbours $=10$, alive cells are from 0 to 9 .

\section{5:- Simulator Design:}

MATLAB stands for Matrix Laboratory, used for scientific computations and is a high-level computing language and interactive surroundings for algorithmic rule development, knowledge visualisation, knowledge anal ysis, and numerical computation. Using MATLAB 7.10.0, varied technical often solved quicker than languages, such as $\mathrm{C}, \mathrm{C}++$, and FORTRAN. Following figures depicts the simulation of coloured brainsegment.jpg image(obtained from MRI) into grey scale image by using MATLAB Tool.

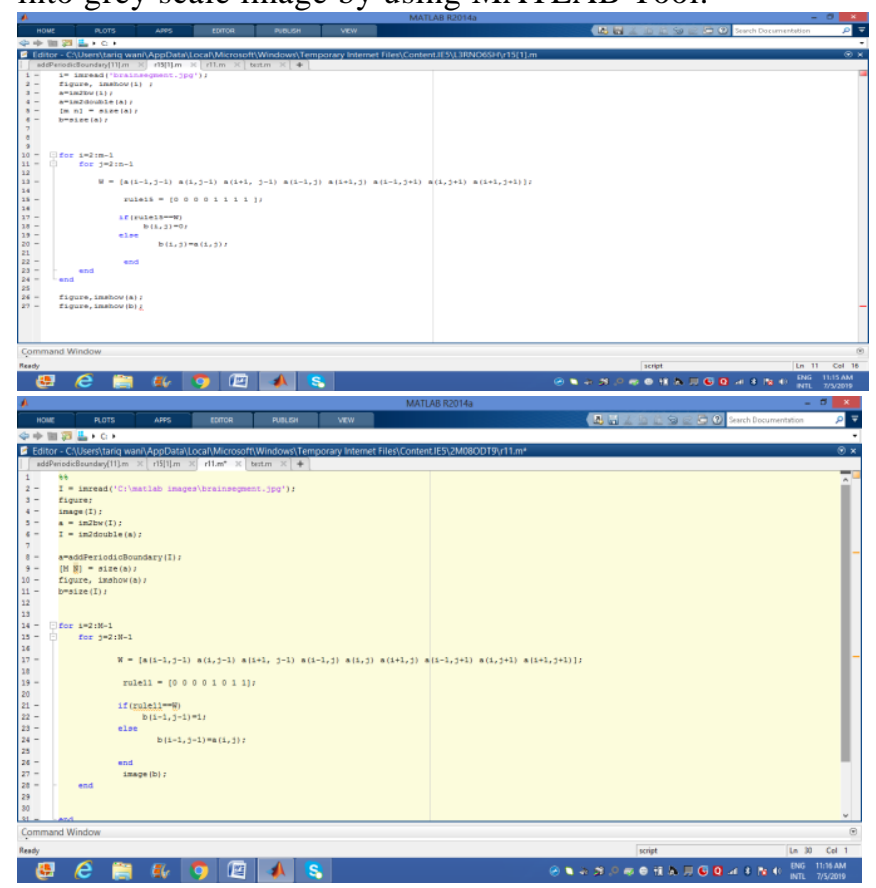

Published By:

Blue Eyes Intelligence Engineering \& Sciences Publication (C) Copyright: All rights reserved.

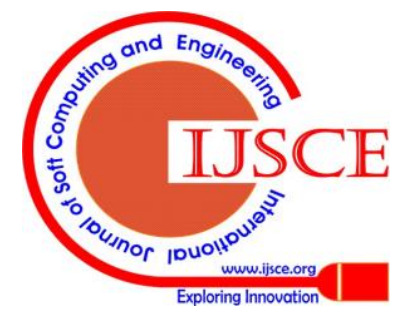


And for edge detection , periodic boundary program is used. In this program we use extended matrix and then fill it with the values.

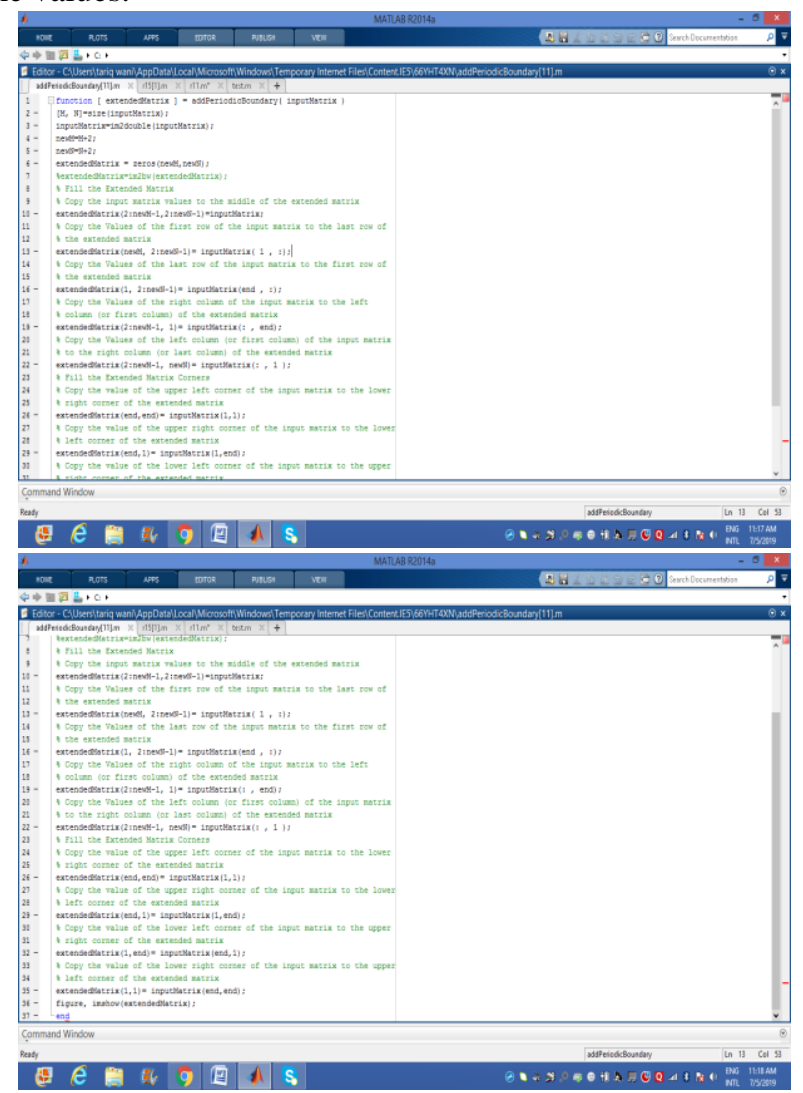

4.6:- Experimental Results:

jpg image is simulated over MATLAB which convert the colored image into gray scale image. Following are the input image as well as its gray scale image:
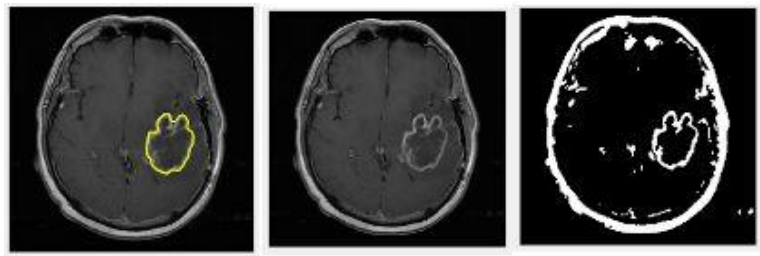

The result of Cellular Automata provides continuous edges with clarity, detecting left vertical line clearly.

In order to get more clarity while taking lesser time, this method of edge detection using cellular automata takes less time in comparison with other methods.

\section{CONCLUSION}

Using different rule of 2D cellular automata (Moore neighborhood), different outputs are obtained. Some outputs are very accurate and clear while some provide blurred and vague outputs. Rules to detect brain tumor by applying edge detection with CA requires few steps, which are very important because our final output depends on them. We have chosen the best method and applied edge detection to gain the favourable result. In this method there is total dependency between neighbourhood pixels, that's the reason of efficiency this algorithm as we are using 2D cellular automata. The final images obtained were clear and efficient.

\section{REFERENCES}

1. Zubair Jeelani, Fasel Qadir,"'Cellular automata-based approach for salt- and - pepper nose filtration”, Journal of King Saud UniversityComputer and information sciences.

2. FaselQadir, Khan K. A, "Investigations of Cellular Automata Linear Rules for Edge Detection", I. J. Computer Network and Information Security, Vol. 3, Pages 47-53, 2012.

3. Shohei Sato and Hitoshi Kanoh, "Evolutionary Design of Edge Detector Using Rule - Changing Cellular Automata", Nature and Biologically Inspired Computing (NaBIC), Second World Congress, Page(s): 60 - 65, 15-17 Dec. 2010.

4. KhadijehMirzaei, HomayunMotameni and RasulEnayatifar, "New method for edge detection and de noising via Fuzzy Cellular Automata”, International Journal of Physical Sciences Volume 6 (13), Pages 3175-3180, 4 July, 2011.

5. ParwinderKaurDhillon, "A Novel framework to Image Edge Detection using Cellular Automata”, IJCA Special Issue on Confluence 2012 - The Next Generation Information Technology Summit Confluence (1):1-5, September 2012.

6. DjemelZiou, Salvatore Tabbone, "Edge Detection Techniques - An Overview", International Journal of Pattern Recognition and Image Analysis, 1998.

7. Leung, CC; Chen, WF; Kwok, PCK; Chan, FHY, "Brain tumor boundary detection in MR image with generalized fuzzy operator", International Conference on Image Processing Proceedings, Barcelona, Spain, Volume 2, Pages 1057-1060, 14-17, 2003.

8. S. Murugavalli and V. Rajamani, "An Improved Implementation of Brain Tumor Detection Using Segmentation Based on Neuro Fuzzy Technique", Journal of Computer Science, Volume 3 (11), Pages 841 846, 2007.

9. Dr. Mahesh Kumar, Manoj Kumar, Mayank Sharma, ’Edge detection for image using cellular automata", International Research Journal of Management Science \& Technology, Vol5Issue 8, year 2014, ISSN 2250-1959.

10. Haralick, Robert M., Digital step edges from Zero crossing of Second Directional Derivatives, Pattern Analysis and Machine Intelligence, IEEE transactions, PAMI6, Issue: 1, Pages 58 - 68, Jan. 1984.

\section{AUTHORS PROFILE}

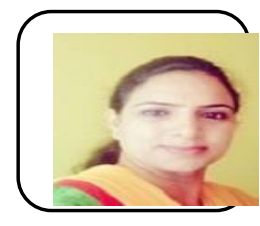

Jasmeena Tariq is a research scholar at, Computer Applications, Bharath Institute of Higher Education and Research, Chennai. Her research interests are Machine Learning, Image Processing, Data mining and Cellular Automata. You can email her at jasmeenat@gmail.com

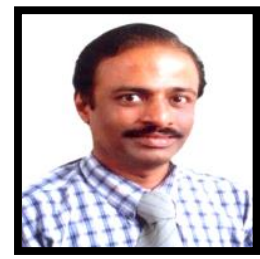

Dr Kumaravel is working as a Professor and Dean, School of Computing, Bharath University, Chennai. His research interest includes Soft Computing, Cloud Computing, Machine Learning, Pervasive Computing and Knowledge Engineering. He is a life Member of ISTE and IET.
Published By:
Blue Eyes Intelligence Engineering \& Sciences Publication

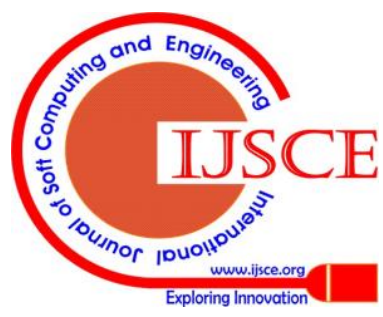

